\title{
Effective-temperature concept: A physical application for nonextensive statistical mechanics
}

\author{
Fernando D. Nobre, ${ }^{1,2, *}$ Andre M. C. Souza, ${ }^{2,3, \dagger}$ and Evaldo M. F. Curado ${ }^{1,2, \ddagger}$ \\ ${ }^{1}$ Centro Brasileiro de Pesquisas Físicas, Rua Xavier Sigaud 150, 22290-180 Rio de Janeiro-RJ, Brazil \\ ${ }^{2}$ National Institute of Science and Technology for Complex Systems, Rua Xavier Sigaud 150, 22290-180 Rio de Janeiro-RJ, Brazil \\ ${ }^{3}$ Departamento de Física, Universidade Federal de Sergipe, 49100-000 São Cristóvão-SE, Brazil
}

(Received 29 June 2012; published 10 December 2012)

\begin{abstract}
The H-theorem [ $(d f / d t) \leqslant 0]$ for a free-energy functional, $f=u-\theta s$ (with $u$ and $s$ representing, respectively, the internal energy and a generalized entropy of a given physical system), has been proven previously by making use of a nonlinear Fokker-Planck equation. Herein we focus on a nonlinear Fokker-Planck equation derived by means of a coarse-graining procedure on the equations of motion of a system of interacting vortices, under overdamped motion, in the absence of thermal noise $(T=0)$. In this case, we show that the parameter $\theta$ is directly related to the density as well as to the interactions among vortices. Generalized quantities such as entropy, internal energy, free energy, and heat capacity are analyzed for varying $\theta$ : important relations and physical behavior analogous to those of standard thermodynamics are found, showing that $\theta$ plays the role of an effective temperature. Estimates of $\theta$ in typical physical situations of different type-II superconductors are presented; in addition to this, possible experimental procedures for varying $\theta$ are proposed.
\end{abstract}

DOI: 10.1103/PhysRevE.86.061113

PACS number(s): 05.40.Fb, 05.45.-a, 05.20.-y, 74.25.Bt

\section{INTRODUCTION}

For the past 100 years, superconducting materials have attracted the attention of many workers from both experimental and theoretical points of view. Most recently, the discovery of high- $T_{c}$ superconductivity has resulted in a renewed interest in these materials due to the possibility of many novel physical applications [1]. These type-II superconductors are characterized by two critical magnetic fields, $B_{c 1}$ and $B_{c 2}$, signaling distinct physical behavior. For applied fields less than the lower critical field $B_{c 1}$, the system presents the Meissner effect, with flux cancellation, in such a way that the entire sample becomes typically a type-I superconductor. Applied fields greater than the upper critical field $B_{c 2}$ result in a complete penetration of the magnetic field, and the material returns to a normal conducting state. However, for field intensities between $B_{c 1}$ and $B_{c 2}$ there is partial penetration of the magnetic field, and the field lines get confined to flux tubes, called vortices [1,2], forming the so-called vortex lattice along which the material presents normal resistivity, whereas its surrounding remains superconducting. These vortices became a paradigm for testing many statistical phenomena due to the recent development of the ability to control their density, interactions, and motion [3-7].

The concept of "effective temperature" is commonly used in physics [8-10]. As examples, one could mention the Fermi temperature $\left(T_{\mathrm{F}}\right)$ in a Fermi-Dirac ideal gas, and the Einstein $\left(T_{\mathrm{E}}\right)$ and Debye $\left(T_{\mathrm{D}}\right)$ temperatures in their respective crystalline-solid models. These temperatures are related to intrinsic properties such as the harmonic frequencies, the mass, and concentrations of particles, leading to well-defined estimates, which represent reference values for each physical system. In the later cases, the effective temperatures $T_{\mathrm{E}}$ and $T_{\mathrm{D}}$

\footnotetext{
*Author to whom all correspondence should be addressed: fdnobre@cbpf.br

†amcsouza@ufs.br

‡evaldo@cbpf.br
}

are usually defined for finite absolute temperatures $(T>0)$, so that distinct physical behaviors are obtained for low temperatures $\left(T \ll T_{\mathrm{E}}, T_{\mathrm{D}}\right)$ and high temperatures $\left(T \gg T_{\mathrm{E}}, T_{\mathrm{D}}\right)$. However, in the case of fermions, the temperature $T_{\mathrm{F}}$ is defined for $T=0$, being directly related to the concentration of electrons, and so it may present large variations depending on the physical system, e.g., $T_{\mathrm{F}} \approx 10^{4} \mathrm{~K}$ for electrons in metals $[8,9]$ or $T_{\mathrm{F}} \approx 10^{9} \mathrm{~K}$ for electrons in white dwarf stars [9]. Furthermore, these examples consist of systems of noninteracting particles, described by Boltzmann-Gibbs (BG) statistical mechanics, according to which certain thermodynamic properties present well-defined behavior for low temperatures, obeying the third-law of thermodynamics, which implies that the entropy per particle, $s_{\mathrm{BG}} \rightarrow 0$, as $T \rightarrow 0$.

The applicability of standard concepts of equilibrium thermodynamics in the study of nonequilibrium states has been considered for many years [11,12]. Out-of-equilibrium physical situations, such as those occurring in glasses [13], chemical reactions $[14,15]$, turbulent fluids, nuclear collisions, and two-temperature systems, among others, are typical examples in which usual definitions were extended, such as thermodynamic potentials, Legendre transformations, and nonequilibrium susceptibilities, and new ones were proposed, such as entropy production. Moreover, the above-mentioned concept of effective temperature has also been introduced in many nonequilibrium systems [16].

One of the most important results in standard nonequilibrium statistical mechanics is the $\mathrm{H}$-theorem $[8,10]$, which guarantees the approach to an equilibrium state. Essentially, this theorem expresses a well-defined sign for the time derivative of the entropy, i.e., $\left(d s_{\mathrm{BG}} / d t\right) \geqslant 0$, in the case of an isolated system, or for the free energy $\left(d f_{\mathrm{BG}} / d t\right) \leqslant 0\left(f_{\mathrm{BG}}=u-T s_{\mathrm{BG}}\right)$, in the case of a system in contact with a heat reservoir. A possible proof of this theorem may be achieved by considering the functional form of $s_{\mathrm{BG}}[P(x, t)]$ in terms of the probability density $P(x, t)$ for finding a particle at a position between $x$ and $x+d x$ in time $t, s_{\mathrm{BG}}[P]=-k \int d x P(x, t) \ln P(x, t)$, and making use of the linear Fokker-Planck equation for the time derivative $\partial P(x, t) / \partial t$. In this way, the $\mathrm{H}$-theorem provides a 
direct connection between the linear Fokker-Planck equation and the BG entropy.

Recently, nonlinear Fokker-Planck equations (NLFPEs) have been widely investigated in the literature [17], motivated by an appropriate description of many complex physical behaviors characterized by anomalous diffusion [18]. Therefore, in the same way that the linear Fokker-Planck equation is associated with normal diffusion and with the BG entropy, the NLFPEs are usually related to anomalous-diffusion phenomena and to generalized entropies (see, e.g., Refs. [19-21] among others). A particular interest has been dedicated to the NLFPE proposed in Ref. [22] related to Tsallis nonextensive statistical mechanics [23-25]. In particular, the $q$-Gaussian distribution, which represents a generalization of the standard Gaussian (recovered in the particular case $q=1$ ) appears naturally from an extremization procedure of the entropy [25], or from the solution of the corresponding nonlinear Fokker-Planck equation [22]. This distribution has been very useful for experiments in many real systems [23,24]; among many, one could highlight the following: (i) the velocities of cold atoms in dissipative optical lattices [26]; (ii) the velocities of particles in quasi-two-dimensional dusty plasma [27]; (iii) the relaxation curves of RKKY spin glasses, such as CuMn and AuFe [28]; (iv) transverse momenta distributions at LEP [29] and LHC experiments [30]; and (v) the overdamped motion of interacting vortices in type-II superconductors [31,32].

In the present work, we will investigate stationary-state properties of the system of interacting vortices of Refs. [31,32]; this study is motivated by a good agreement found between the vortex-position stationary-state distributions obtained by means of molecular-dynamics simulations and the analytical solution of the associated NLFPE [31]. In the next section we define the system, the NLFPE, and its related entropy. In Sec. III, we explore the effective-temperature concept by showing that it satisfies important relations and physical properties, playing a role very similar to that of the absolute temperature in standard thermodynamics. Finally, in Sec. IV we present our conclusions.

\section{THE PHYSICAL SYSTEM AND ITS ASSOCIATED NONLINEAR FOKKER-PLANCK EQUATION}

The following model of interacting vortices has been used in the literature to represent flux lines in disordered type-II superconductors (see, e.g., Refs. [33,34]); the equation of motion of a flux line $i$ under overdamped motion [i.e., with $\left(d \mathbf{v}_{i} / d t\right)=0$ ], in a medium with an effective friction coefficient $\eta$, may be written as

$$
\eta \mathbf{v}_{i}=\mathbf{F}_{i}^{\mathrm{pp}}+\mathbf{F}_{i}^{\mathrm{ext}} \quad(i=1,2, \ldots, N) .
$$

In the equation above, $\mathbf{v}_{i}$ represents the velocity, whereas the terms on the right-hand side depict the forces acting on flux line $i$. The first contribution, $\mathbf{F}_{i}^{\mathrm{pp}}$, takes into account the interactions among vortices [each vortex interacts with the remaining $(N-1)$ vortices], whereas $\mathbf{F}_{i}^{\text {ext }}$ represents an external force acting on vortex $i$. We consider

$$
\mathbf{F}_{i}^{\mathrm{pp}}=\frac{1}{2} \sum_{j \neq i} B^{\mathrm{pp}}\left(r_{i j}\right) \hat{\mathbf{r}}_{i j}, \quad B^{\mathrm{pp}}\left(r_{i j}\right)=f_{0} K_{1}\left(r_{i j} / \lambda\right),
$$

where $r_{i j}=\left|\mathbf{r}_{i}-\mathbf{r}_{j}\right|$ stands for the distance between vortices $i$ and $j$, and $\hat{\mathbf{r}}_{i j}=\left(\mathbf{r}_{i}-\mathbf{r}_{j}\right) / r_{i j}$ is a vector defined along the axis joining them. Moreover, $K_{1}$ represents a modified Bessel function of the second kind of order 1 and $f_{0}$ is a positive constant. These interactions are defined in terms of a characteristic length scale $\lambda$, known as the London penetration length; other lengths of this system will be measured in units of $\lambda$. The vortex-vortex interactions $B^{\mathrm{pp}}\left(r_{i j}\right)$ are repulsive and radially symmetric, whereas the external forces $\mathbf{F}_{i}^{\text {ext }}$ should be associated with a confining type of potential, so that the system can reach a stationary state after a sufficiently long time. Herein this problem will be considered in a two-dimensional box of dimensions $L_{x}$ and $L_{y}$ to conform with previous numerical simulations [31,32].

Considering an external force in the $x$ direction, $\mathbf{F}^{\text {ext }}=$ $-A(x) \hat{\mathbf{x}}$, a coarse-graining procedure in Eq. (1) leads to the following NLFPE [31,32,34]:

$$
\begin{aligned}
\eta \frac{\partial P(x, t)}{\partial t}= & -\frac{\partial[A(x) P(x, t)]}{\partial x} \\
& +2 D \frac{\partial}{\partial x}\left\{[\lambda P(x, t)] \frac{\partial P(x, t)}{\partial x}\right\},
\end{aligned}
$$

where $D=N \pi f_{0} \lambda^{2} / L_{y}$. It should be mentioned that this equation represents a particular case of the NLFPE introduced in Ref. [22], which contains a more general diffusion term, defined by replacing $2 D[\lambda P(x, t)] \rightarrow \nu D[\lambda P(x, t)]^{\nu-1}$, where $v$ is a real number; Eq. (3) corresponds to the particular case $v=2$. Moreover, similarly to the linear Fokker-Planck equation [10], the first term on the r.h.s. carries the contribution of the external potential, whereas the second one comes from the interaction of a single flux line with the medium, and it is usually associated with an effective temperature; herein, this later term represents the effect of $N-1$ vortices on the tagged vortex. Therefore, the distribution $P(x, t)$ will refer to one flux line of the above-mentioned system, and so all physical quantities to be derived from this distribution will correspond to one-vortex properties.

The time-dependent solution of the general NLFPE was found for a harmonic external force, $A(x)=-\alpha x(\alpha>0)$, and the initial condition $P(x, 0)=\delta(x)$ [22],

$$
P(x, t)=B(t)\left[1+b(t)(1-v) x^{2}\right]_{+}^{1 /(v-1)},
$$

where $[u]_{+}=u$ for $u>0$, zero otherwise, and the timedependent coefficients $B(t)$ and $b(t)$ are related to each other in order to preserve the normalization of $P(x, t)$ for all times $t$. The particular case of interest herein corresponds to $v=2$,

$$
P(x, t)=B(t)\left[1-b(t) x^{2}\right]_{+},
$$

presenting a compact support in the interval $[-\bar{x}(t), \bar{x}(t)]$, with $\bar{x}(t)=b^{-1 / 2}(t)$.

The H-theorem may be proved using Eq. (3) by imposing a well-defined sign for the time derivative of the one-vortex free-energy functional [31,32],

$$
f[P]=u[P]-\theta s[P], \quad u[P]=\int_{-\bar{x}(t)}^{\bar{x}(t)} d x \phi(x) P(x, t),
$$

where $\theta$ is a positive quantity with units of temperature and $\phi(x)$ represents the external potential $[A(x)=-d \phi(x) / d x]$. 
TABLE I. Estimates of the energy in Eq. (11), as well as of the associated effective temperature $\theta$, for typical type-II superconductors at the lower critical field $B_{c 1}$. The values of the London penetration length $\lambda$ and the dimensionless parameter $\kappa(\kappa=\lambda / \xi$ denotes the ratio between the penetration and coherence lengths, respectively) were taken from Ref. [1]. The values of the mean distance between vortices $d$, as well as of the density of vortices $n$, were calculated as described in the text.

\begin{tabular}{lccccrrr}
\hline \hline Type-II superconductor & $\lambda(\mathrm{nm})$ & $\kappa$ & $d / \lambda$ & $d(\mathrm{~nm})$ & $n\left(10^{9}\right.$ vortices $\left./ \mathrm{m}\right)$ & $k \theta\left(10^{-15} \mathrm{~J}\right)$ & $\theta\left(10^{8} \mathrm{~K}\right)$ \\
\hline $\mathrm{Nb}$ & 50 & 1.28 & 7.67 & 383.41 & 6.8 & 24.0 & 17.0 \\
$\mathrm{~Pb}-\mathrm{Bi}$ & 200 & 10 & 2.51 & 502.16 & 4.0 & 3.4 \\
$\mathrm{Nb}-\mathrm{N}$ & 200 & 40 & 1.98 & 396.74 & 6.4 & 5.5 & 4.5 \\
$\mathrm{YBa}_{2} \mathrm{Cu}_{3} \mathrm{O}_{7}$ & 170 & 95 & 1.79 & 303.52 & 11.0 & 10.0 \\
\hline \hline
\end{tabular}

As usual, the internal energy is defined solely in terms of the external potential; the contribution of the interactions among vortices will appear in the parameter $\theta$. To satisfy the $\mathrm{H}$-theorem, the associated entropy should be given by $[31,32]$

$$
s[P]=k\left\{1-\lambda \int_{-\bar{x}(t)}^{\bar{x}(t)} d x[P(x, t)]^{2}\right\}
$$

and we have identified $k \theta=D$.

Indeed, some of the above-mentioned results follow readily if one writes Eq. (3) in the form [17]

$$
\eta \frac{\partial P(x, t)}{\partial t}=\frac{\partial}{\partial x}\left[P(x, t) \frac{\partial}{\partial x}\left(\frac{\delta f[P]}{\delta P(x, t)}\right)\right]
$$

where $\delta f[P] / \delta P(x, t)$ denotes a functional derivative with respect to the probability $P(x, t)$, and the definition $D=k \theta$ becomes necessary. In this way, the $\mathrm{H}$-theorem becomes

$$
\begin{aligned}
\frac{d f}{d t} & =\int_{-\bar{x}(t)}^{\bar{x}(t)} d x[\phi(x)+2 k \theta \lambda P(x, t)] \frac{\partial P(x, t)}{\partial t} \\
& =\frac{1}{\eta} \int_{-\bar{x}(t)}^{\bar{x}(t)} d x \frac{\delta f[P]}{\delta P(x, t)} \frac{\partial}{\partial x}\left[P(x, t) \frac{\partial}{\partial x}\left(\frac{\delta f[P]}{\delta P(x, t)}\right)\right],
\end{aligned}
$$

in which we have substituted Eq. (8). Now, integrating by parts and using the property for the distribution of Eq. (5), $\left.P(x, t)\right|_{x= \pm \bar{x}(t)}=0$, one has

$$
\frac{d f}{d t}=-\frac{1}{\eta} \int_{-\bar{x}(t)}^{\bar{x}(t)} d x P(x, t)\left[\frac{\partial}{\partial x}\left(\frac{\delta f[P]}{\delta P(x, t)}\right)\right]^{2} \leqslant 0 .
$$

One should notice that the distribution of Eq. (5) coincides with the one obtained through an extremization of the above entropy, considering the constraint for probability normalization, in addition to the constraint of Eq. (6) for the internal energy, with $\theta$ being a Lagrange multiplier. This distribution may be recognized as the particular case $q=0$ of a
$q$-Gaussian that appears frequently in nonextensive statistical mechanics [cf. Eq. (4) with $v=2-q$ ]. The "duality" $q \leftrightarrow$ $(2-q)$ between the distribution index $q=0$ and the entropic exponent that appears in Eq. (7) occurs whenever the entropy is extremized by considering the energy definition in Eq. (6); equal indexes appear only when one uses a generalized definition for the internal energy [23].

\section{THE EFFECTIVE-TEMPERATURE CONCEPT}

Now, considering the thermodynamic limit as $N, L_{y} \rightarrow \infty$, keeping the ratio $n=\left(N / L_{y}\right)$ finite, the Lagrange multiplier $\theta$ introduced in Eq. (6) is obtained,

$$
k \theta=\lim _{N, L_{y} \rightarrow \infty} \frac{N \pi f_{0} \lambda^{2}}{L_{y}}=n \pi f_{0} \lambda^{2} .
$$

One should recall that the pinning strength is given by $f_{0}=\Phi_{0}^{2} /\left(8 \pi^{2} \lambda^{3}\right)$ [1], where $\Phi_{0}=h /(2 e)$ represents the unit of flux quantization; in this way, the above quantity $k \theta$ exhibits dimensions of energy and is directly related to the interactions between vortices, being always positive; moreover, $\theta$ corresponds to an "effective temperature" for the present system. Since distinct materials are characterized by different penetration lengths $\lambda$ (see Tables I and II), the pinning strength may be altered only by changing the material. However, for a given material, the density of vortices $n$ may be varied either by changing the external magnetic field $B_{\text {ext }}$ in the range $B_{c 1} \leqslant B_{\text {ext }} \leqslant B_{c 2}$, or by applying an alternating electrical current [3-6]; within this later procedure, one may even eliminate all vortices, yielding the desirable limit $n \rightarrow 0$. Therefore, any proposal for varying $\theta$ experimentally should be directly related to an adequate control of the vortex density.

In Tables I and II, we estimate values for the energy $k \theta$ as well as of the associated temperature $\theta$ (herein we consider $k$ as the Boltzmann constant) for typical type-II superconductors,

TABLE II. Estimates of the energy in Eq. (11), as well as of the associated effective temperature $\theta$, for typical type-II superconductors at the upper critical field $B_{c 2}$. The values of the London penetration length $\lambda$ and the dimensionless parameter $\kappa(\kappa=\lambda / \xi$ denotes the ratio between the penetration and coherence lengths, respectively) were taken from Ref. [1]. The values of the mean distance between vortices $d$, as well as of the density of vortices $n$, were calculated as described in the text.

\begin{tabular}{lcccccc}
\hline \hline Type-II superconductor & $\lambda(\mathrm{nm})$ & $\kappa$ & $d / \lambda$ & $d(\mathrm{~nm})$ & $n($ vortices $/ \mathrm{m})$ & $k \theta(\mathrm{J})$ \\
\hline $\mathrm{Nb}$ & 50 & 1.28 & 2.10 & 105 & $9.0 \times 10^{10}$ & $3.0 \times 10^{-13}$ \\
$\mathrm{~Pb}-\mathrm{Bi}$ & 200 & 10 & 0.27 & 54 & $3.4 \times 10^{11}$ & $2.9 \times 10^{-13}$ \\
$\mathrm{Nb}-\mathrm{N}$ & 200 & 40 & 0.0675 & 13.5 & $5.5 \times 10^{12}$ & $4.6 \times 10^{-12}$ \\
$\mathrm{YBa}_{2} \mathrm{Cu}_{3} \mathrm{O}_{7}$ & 170 & 95 & 0.0284 & 4.8 & $4.3 \times 10^{13}$ & $4.2 \times 10^{10}$ \\
\hline \hline
\end{tabular}


at both lower (Table I) and upper (Table II) critical fields. For that, we used the relations [1]

$$
\left\langle B_{\text {in }}\right\rangle=B_{c 1} \frac{8 \pi \lambda^{2}}{\sqrt{3} d^{2} \ln \kappa}, \quad B_{c 2}=B_{c 1} \frac{2 \kappa^{2}}{\ln \kappa},
$$

where $d$ represents the mean distance between vortices, and the dimensionless quantity $\kappa=\lambda / \xi$ (with $\kappa>1$ for type-II superconductors) denotes the ratio between the two relevant lengths of a type-II superconductor, namely the penetration and coherence lengths, respectively. By setting the average internal field $\left\langle B_{\text {in }}\right\rangle$ equal to either $B_{c 1}$ or $B_{c 2}$, we have estimated the mean distance between vortices in these two limiting cases. Then, considering a vortex lattice of size $L_{x} \times L_{y}$ (in real systems, these dimensions are of the order of a few $\mathrm{mm}$ [7]), one may estimate the ratio $n=N / L_{y} \approx L_{x} L_{y} /\left(d^{2} L_{y}\right)$, i.e., $n \approx L_{x} / d^{2}$; the corresponding values are presented in Tables I and II for a vortex lattice of a typical linear size $L_{x}=1 \mathrm{~mm}$.

Comparing these two tables, one notices that the density of vortices, and consequently the energy $k \theta$, increases with the external magnetic field, leading to variations in the associated values of $\theta$ in the range $10^{8}-10^{12} \mathrm{~K}$; these estimates should be compared with the Fermi temperature for electrons in white dwarf stars ( $\left.T_{\mathrm{F}} \approx 10^{9} \mathrm{~K}[9]\right)$. The main difference is that in the present case one has the possibility of decreasing the density of vortices experimentally by applying an alternating electrical current, as described in Refs. [3-6]. One should recall that herein we are neglecting the effects of thermal noise (i.e., $T=0$ ), which appears to be a good approximation when considering the order of magnitudes of $\theta$. Moreover, possible experimental realizations have to occur for $T \ll \theta$, so that thermal effects should not influence those associated with the energy $k \theta$; the estimates in Tables I and II suggest that measurements of some of the properties described below can be carried even at room temperature.

In what follows, we will analyze the behavior of important physical properties of the system as the effective temperature $\theta$ varies; we will show that $\theta$ plays a role very similar to the absolute temperature in standard thermodynamics. One should recall that certain quantities, such as entropy and specific heat, become zero for $T=0$ in BG statistical mechanics; however, in Ref. [31], a curious situation concerning the third law of thermodynamics was verified, where $s_{\mathrm{BG}} \rightarrow 0$, keeping a generalized entropic form $s>0$, as $T \rightarrow 0$. Herein, we will analyze generalized forms of these quantities, related to the entropic form of Eq. (7), showing that they present a nontrivial behavior for varying $\theta$, being different from zero even for zero thermal noise. For convenience, herein these quantities will be referred to by means of the same nomenclature used in standard thermodynamics.

Let us then introduce the dimensionless variable,

$$
\tau=\frac{k \theta}{\alpha \lambda^{2}}=\frac{n \pi f_{0}}{\alpha}
$$

which corresponds to the ratio between the effective thermal energy $k \theta$ (associated with the repulsive interactions among vortices) and a typical confining harmonic energy, $\alpha \lambda^{2}$. Since this confining energy is responsible for the system reaching its stationary state, one should have $k \theta \sim \alpha \lambda^{2}$ in such a way that all important physical phenomena are expected to occur for $\tau \sim O(1)$; this gives further support for the introduction of the variable in Eq. (13).

The stationary-state solution of Eq. (3) may be found easily [31],

$P_{\mathrm{st}}(x)=\frac{\alpha}{4 D \lambda}\left(x_{e}^{2}-x^{2}\right)=\frac{1}{4 \tau \lambda}\left[\left(\frac{x_{e}}{\lambda}\right)^{2}-\left(\frac{x}{\lambda}\right)^{2}\right],|x|<x_{e}$,

where $x_{e}=\lim _{t \rightarrow \infty} \bar{x}(t)=\lim _{t \rightarrow \infty} b^{-1 / 2}(t)$. The normalization condition for $P_{\mathrm{st}}(x)$ yields $x_{e}=(3 D \lambda / \alpha)^{1 / 3}$, or in terms of the variable $\tau, x_{e}=(3 \tau)^{1 / 3} \lambda$. Its even moments can be calculated,

$$
\begin{aligned}
\left\langle x^{2 l}\right\rangle & =\int_{-x_{e}}^{x_{e}} d x x^{2 l} P_{\mathrm{st}}(x) \\
& =\frac{3}{(2 l+1)(2 l+3)}(3 \tau)^{2 l / 3} \lambda^{2 l} \quad(l=0,1,2, \ldots),
\end{aligned}
$$

from which one obtains the special case $l=1$,

$$
\left\langle x^{2}\right\rangle=\frac{1}{5}(3 \tau)^{2 / 3} \lambda^{2} \Rightarrow\left\langle x^{2}\right\rangle \sim \theta^{2 / 3} .
$$

The anomalous diffusion, obtained previously from a dynamical analysis of this system, i.e., $\left\langle x^{2}\right\rangle \sim t^{2 / 3}$ [31,32], together with the above result suggest that $\left\langle x^{2}\right\rangle \sim(\theta t)^{2 / 3}$ (this is to be compared with $\left\langle x^{2}\right\rangle \sim T t$, valid in standard diffusion [8]).

The distribution $P_{\mathrm{st}}(x)$ is exhibited versus $x$ (in units of $\lambda$ ) in Fig. 1 for typical values of $\tau$, where one sees the spreading of the distribution as $\tau$ increases, following Eq. (16); one should recall the similarity of this effect with normal diffusion as the temperature $T$ increases. In the inset, we present $P_{\mathrm{st}}(x)$ for the special value $\tau^{*}=0.072$, leading to a very narrow distribution; as will be shown next, $\tau^{*}$ corresponds to the value at which the entropy becomes zero.

The knowledge of the equilibrium distribution in Eq. (14) allows for the computation of one-vortex physical properties, such as those in Eqs. (6) and (7). For the internal energy,

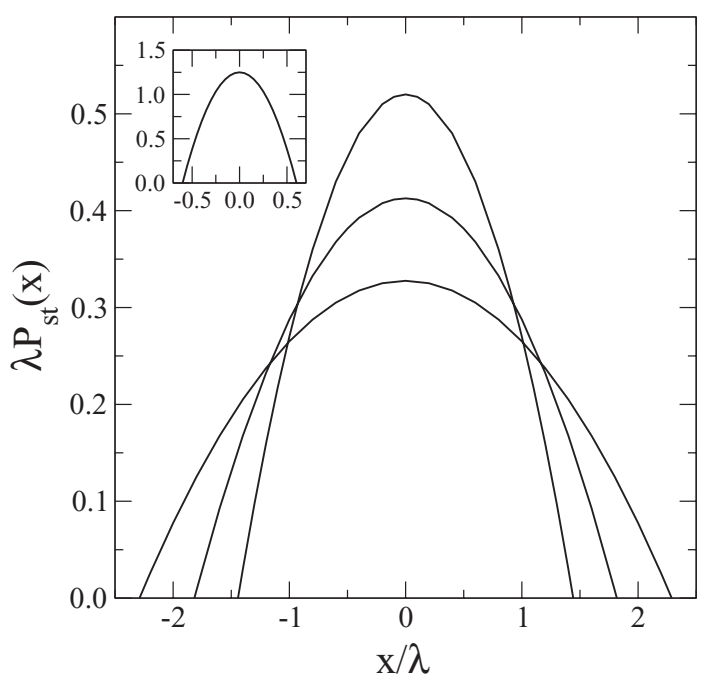

FIG. 1. The dimensionless equilibrium distribution $\lambda P_{\mathrm{st}}(x)$ is represented vs the position $x$ (in units of $\lambda$ ) for typical choices of $\tau$, namely $\tau=1,2,4$ (from top to bottom). In the inset, we present the same distribution in the special value $\tau^{*}=0.072$, for which the entropy becomes zero, in a different scale. 


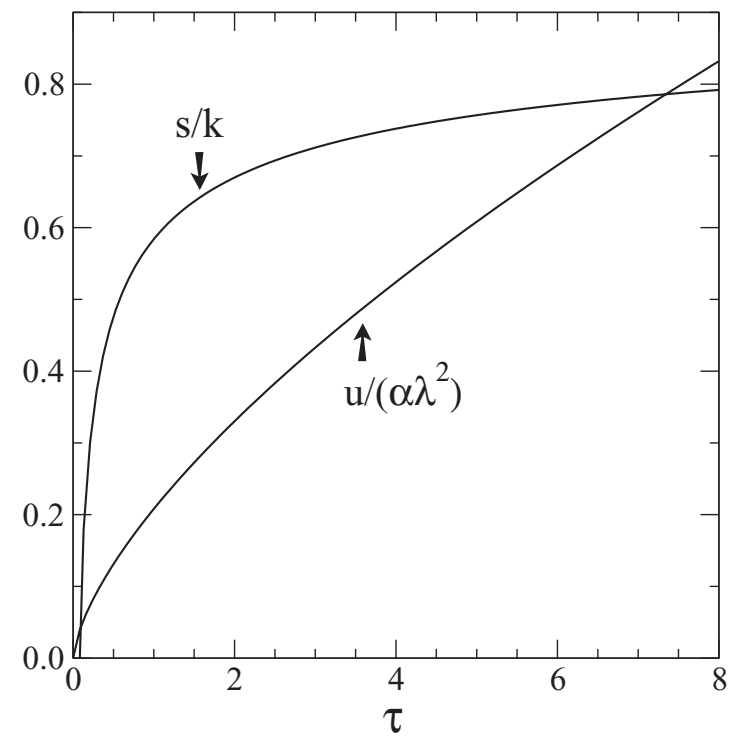

FIG. 2. The internal energy $u$ (in units of $\alpha \lambda^{2}$ ) and the entropy $s$ (in units of $k$ ) are represented vs the dimensionless parameter $\tau$. The entropy becomes negative only for very small values of $\tau$, namely for $\tau<0.072$.

one has

$$
u=\int_{-x_{e}}^{x_{e}} d x \frac{\alpha x^{2}}{2} P_{\mathrm{st}}(x)=\frac{1}{10} \alpha x_{e}^{2}=\frac{3^{2 / 3}}{10} \alpha \lambda^{2} \tau^{2 / 3},
$$

which is shown versus $\tau$ in Fig. 2. One notices a monotonic increase of $u$ with $\tau$, as well as a curvature, which are very similar to those of the internal energy of physical systems for increasing the absolute temperature $T$ [8-10]. From Eq. (7), one obtains the entropy

$$
s=k\left[1-\frac{1}{5}\left(\frac{9}{\tau}\right)^{1 / 3}\right]
$$

as represented in Fig. 2. The entropy above may be written also in terms of the internal energy of Eq. (17),

$$
s(u)=k\left[1-\frac{3}{5}\left(\frac{\alpha \lambda^{2}}{10 u}\right)^{1 / 2}\right],
$$

where one notices that $s$ is a concave function of $u$, as thermodynamically expected. An interesting point concerns the negative-entropy anomaly, i.e., the entropy becomes negative for sufficiently small values of $\tau$, or equivalently, for small $u /\left(\alpha \lambda^{2}\right)$. In fact, one obtains that $s=0$ for $\tau^{*}=(9 / 125)=$ 0.072 ; this corresponds to $u^{*}=(9 / 250) \alpha \lambda^{2}=0.036 \alpha \lambda^{2}$ as well as to $x_{e}=0.6 \lambda$. In fact, for $\tau<\tau^{*}$ one gets a very narrow $P_{\mathrm{st}}(x)$, for which all vortices get confined in a small region (typically of order $\lambda$ ), as shown in the inset of Fig. 1, with each vortex feeling strongly the presence of the remaining ones; clearly in this case, a quantum-mechanical treatment should be used instead. Hence, the threshold $\tau^{*}$ signals the region of validity of the present classical results. This anomaly is similar to those appearing in standard statistical mechanics of classical systems, where the entropy becomes negative for sufficiently low values of $T$; a typical analogous situation occurs in the classical ideal gas when the mean distance between molecules becomes of the order of the de Broglie wavelength [8-10]. Therefore, one can define two distinct physical regimes associated with the variable $\tau$ : (i) the quantum regime $\left(\tau \lesssim \tau^{*}\right)$, where the confining potential dominates, leading to a narrow $P_{\mathrm{st}}(x)$; (ii) the classical regime $\left(\tau \gg \tau^{*}\right)$, where one finds vortices far apart from each other, due to strong repulsion effects, yielding broad stationary distributions. The present analysis applies to the latest situation.

From Eq. (19) one verifies analytically the fundamental relation

$$
\frac{\partial s(u)}{\partial u}=\frac{1}{\theta},
$$

showing that $\theta$ plays a role very similar to the absolute temperature of standard thermodynamics. In addition to this, one may calculate the free energy of Eq. (6)

$$
f=\alpha \lambda^{2}\left[\frac{3^{5 / 3}}{10} \tau^{2 / 3}-\tau\right]
$$

which satisfies $(\partial f) /(\partial \theta)=-s$, yielding additional support for the interpretation of $\theta$ as an effective temperature.

The specific heat may be calculated in three different ways, namely from the internal energy, or the entropy, or the free energy; such calculations lead to

$$
c=\frac{\partial u}{\partial \theta}=\theta \frac{\partial s}{\partial \theta}=-\theta \frac{\partial^{2} f}{\partial^{2} \theta}=\frac{3^{2 / 3}}{15} k \tau^{-1 / 3} .
$$

Notice that $c$ diverges for $\theta \rightarrow 0$, revealing the unphysical behavior signaled by the negative entropy; indeed, for $\tau=\tau^{*}$ one has $c=k / 3$, which represents its maximum acceptable value. The specific heat above is directly related to energy fluctuations,

$$
\left\langle E^{2}\right\rangle-\langle E\rangle^{2}=\frac{18}{7} \theta^{2} c^{2}, \quad\left\langle E^{2}\right\rangle=\int_{-\bar{x}(t)}^{\bar{x}(t)} d x \phi^{2}(x) P(x, t),
$$

leading to the expected result $\left\langle E^{2}\right\rangle \geqslant\langle E\rangle^{2}$ [herein $\langle E\rangle \equiv u$, as defined in Eq. (6)]. Since in the present application one has $q=0$, the above relation may be written as $\left\langle E^{2}\right\rangle-\langle E\rangle^{2} \propto$ $(k \theta)^{2}(c / k)^{2-q}$, suggesting a generalization of the well-known relation $\left\langle E^{2}\right\rangle-\langle E\rangle^{2}=k_{\mathrm{B}} T^{2} c$.

Furthermore, the results above are fully compatible with the definition of a partition function [23,35],

$$
\begin{aligned}
& f_{2-q}=-\frac{1}{\beta} \ln _{2-q} Z_{2-q}, \quad u_{2-q}=-\frac{\partial}{\partial \beta} \ln _{2-q} Z_{2-q}, \\
& \left(\ln _{2-q} x=\frac{x^{q-1}-1}{q-1}\right),
\end{aligned}
$$

where we have defined $\beta=1 /(k \theta)$; the partition function in the present case $(q=0)$ is given by

$$
Z_{2}=\frac{10}{3^{5 / 3}} \tau^{1 / 3}
$$

\section{CONCLUSIONS}

We have presented the concept of an effective temperature $\theta$ for a system of interacting vortices under overdamped motion in the absence of thermal noise $(T=0)$. This 
quantity is directly related to the density as well as to the interactions among vortices, being always positive by definition. Generalized thermodynamic functions, within the framework of nonextensive statistical mechanics, such as entropy, internal energy, free energy, and specific heat, have been studied for varying this effective temperature. Apart from preserving important thermodynamic relations in terms of $\theta$, these quantities presented a behavior very similar to the corresponding ones found in standard thermodynamics, as one changes the absolute temperature $T$.

The present system appears naturally as a typical physical application for nonextensive statistical mechanics; the predictive power of the results above could be tested by means of adequate experiments in type-II superconductors under an appropriate confining potential. Through the experimental techniques available nowadays, which allow changes in the density of vortices and consequently lead to variations in $\theta$, some of the properties discussed above will hopefully be measured. Other physical systems, characterized by repulsive particle-particle interactions decaying with distance, may also be described by the same nonlinear Fokker-Planck equation, with a different value for the diffusion parameter $D$; these systems should exhibit properties similar to those presented herein.

\section{ACKNOWLEDGMENTS}

We thank Constantino Tsallis for frutiful conversations. Partial financial support from CNPq, CAPES, and FAPERJ (Brazilian agencies) is acknowledged.
[1] C. P. Poole Jr., H. A. Farach, and R. J. Creswick, Superconductivity (Academic, London, 1995).

[2] G. Blatter, M. V. Feigel'man, V. B. Gerhkenbein, A. I. Larkin, and V. M. Vinokur, Rev. Mod. Phys. 66, 1125 (1994).

[3] C.-S. Lee, B. Jankó, I. Derényi, and A.-L. Barabási, Nature (London) 400, 337 (1999).

[4] I. Derényi, Appl. Phys. A 75, 217 (2002).

[5] J. E. Villegas, S. Savel'ev, F. Nori, E. M. Gonzalez, J. V. Anguita, R. García, and J. L. Vicent, Science 302, 1188 (2003).

[6] B. Y. Zhu, F. Marchesoni, and F. Nori, Phys. Rev. Lett. 92, 180602 (2004)

[7] I. Fridman, C. Kloc, C. Petrovic, and J. Y. T. Wei, Appl. Phys. Lett. 99, 192505 (2011).

[8] F. Reif, Fundamentals of Statistical and Thermal Physics (McGraw-Hill, New York, 1965).

[9] H. B. Callen, Thermodynamics, 2nd ed. (Wiley, New York, 1985).

[10] L. E. Reichl, A Modern Course in Statistical Physics, 2nd ed. (Wiley, New York, 1998).

[11] I. Prigogine, Introduction of the Thermodynamics of Irreversible Processes (Wiley, New York, 1967).

[12] S. R. de Groot and P. Mazur, Non-Equilibrium Thermodynamics (Dover, New York, 1984).

[13] Th. M. Nieuwenhuizen, Phys. Rev. Lett. 80, 5580 (1998).

[14] M. Grmela, Adv. Chem. Eng. 39, 75 (2010).

[15] M. Grmela, Physica D 241, 976 (2012).

[16] J. Casas-Vázquez and D. Jou, Rep. Prog. Phys. 66, 1937 (2003).

[17] T. D. Frank, Nonlinear Fokker-Planck Equations: Fundamentals and Applications (Springer, Berlin, 2005).

[18] J. P. Bouchaud and A. Georges, Phys. Rep. 195, 127 (1990).

[19] M. Shiino, J. Math. Phys. 42, 2540 (2001); T. D. Frank and A. Daffertshofer, Physica A 295, 455 (2001); P. H. Chavanis, Phys. Rev. E 68, 036108 (2003).
[20] V. Schwämmle, F. D. Nobre, and E. M. F. Curado, Phys. Rev. E 76, 041123 (2007); V. Schwämmle, E. M. F. Curado, and F. D. Nobre, Eur. Phys. J. B 58, 159 (2007).

[21] V. Schwämmle, E. M. F. Curado, and F. D. Nobre, Eur. Phys. J. B 70, 107 (2009).

[22] A. R. Plastino and A. Plastino, Physica A 222, 347 (1995); C. Tsallis and D. J. Bukman, Phys. Rev. E 54, R2197 (1996).

[23] C. Tsallis, Introduction to Nonextensive Statistical Mechanics (Springer, New York, 2009).

[24] C. Tsallis, Braz. J. Phys. 39, 337 (2009).

[25] C. Tsallis, J. Stat. Phys. 52, 479 (1988).

[26] P. Douglas, S. Bergamini, and F. Renzoni, Phys. Rev. Lett. 96, 110601 (2006).

[27] B. Liu and J. Goree, Phys. Rev. Lett. 100, 055003 (2008).

[28] R. M. Pickup, R. Cywinski, C. Pappas, B. Farago, and P. Fouquet, Phys. Rev. Lett. 102, 097202 (2009).

[29] I. Bediaga, E. M. F. Curado, and J. M. de Miranda, Physica A 286, 156 (2000).

[30] V. Khachatryan et al. (CMS Collaboration), Phys. Rev. Lett. 105, 022002 (2010).

[31] J. S. Andrade Jr., G. F. T. da Silva, A. A. Moreira, F. D. Nobre, and E. M. F. Curado, Phys. Rev. Lett. 105, 260601 (2010).

[32] M. S. Ribeiro, F. D. Nobre, and E. M. F. Curado, Phys. Rev. E 85, 021146 (2012).

[33] H. J. Jensen, A. Brass, and A. J. Berlinsky, Phys. Rev. Lett. 60, 1676 (1988); O. Pla and F. Nori, ibid. 67, 919 (1991); R. A. Richardson, O. Pla, and F. Nori, ibid. 72, 1268 (1994).

[34] S. Zapperi, A. A. Moreira, and J. S. Andrade, Phys. Rev. Lett. 86, 3622 (2001).

[35] E. M. F. Curado and C. Tsallis, J. Phys. A 24, L69 (1991); Corrigendum 25, 1019 (1992). 